\title{
Construindo a relação entre Funções Executivas e Mecânicas de Jogos Digitais
}

\author{
Katiane K. G. Krause ${ }^{1}$, Isabela Gasparini, ${ }^{1,2}$, Marcelo da Silva Hounsell ${ }^{2}$ \\ ${ }^{1}$ Programa de Pós-Graduação em Ensino de Ciências, Matemática e Tecnologias - \\ PPGECMT e ${ }^{2}$ Programa de Pós-Graduação em Computação Aplicada - PPGCA \\ Universidade do Estado de Santa Catarina (UDESC) - Joinville, SC, Brasil \\ kati.kazuza@gmail.com, \{isabela.gasparini, marcelo.hounsell\}@udesc.br
}

\begin{abstract}
Executive functions are cognitive abilities related to school success and behavior management. Based on the systematic review process 32 works that involving the study of the impact of digital games on executive functions, construction and validation of the evaluation instruments and training through digital games have been identified. This review was able to identify 22 digital game mechanics to used develop executive components. Although none of the games were educational ones, nine were aimed at to promoting executive skills in scholars. This research shows that game mechanics are game elements capable of explaining how digital games develop executive functions.
\end{abstract}

Resumo. As funções executivas são habilidades cognitivas relacionadas com o sucesso escolar e gestão do comportamento. Baseado no processo de revisão sistemática, identificou-se 32 pesquisas envolvendo o estudo do impacto dos jogos digitais nas funções executivas, construção e validação de instrumento de avaliação e treinamento por meio de jogos digitais. Foram delimitadas 22 mecânicas de jogos digitais utilizadas para desenvolver componentes executivos. Apesar de nenhum dos estudos serem jogos educacionais (ensino de conteúdo), 9 estavam voltados para promoção de habilidades executivas em escolares. Esta pesquisa mostra que mecânicas do jogo são elementos de jogos digitais que desenvolvem as funções executivas.

\section{Introdução}

Desenvolver soluções, decidir, aguardar a vez de fala, manter-se focado, executar cálculos, aprender segunda língua ou estudar são tarefas de alta complexidade para o cérebro, pois requerem autocontrole e gerenciamento da atenção [Katz, 2008]. Por volta dos três anos de idade e com pico de maturação entre os cinco e sete anos, nosso cérebro desenvolve a capacidade de gerir nossas ações de maneira menos impulsiva e reativa, possibilitando melhor autocontrole durante a execução de tarefas [Diamond, 2013]. Todavia, ser capaz não significa ser hábil, estando nosso desempenho atrelado ao tipo e frequência das experiências a que nos expomos [Kuhn, 2006]. Baixa habilidade nessas funções está associada a condições de abuso de substâncias, agressividade, improdutividade e volatilidade no emprego [Zelazo, 2016]. Ademais, considera-se o sucesso escolar mais dependente das funções executivas do que o quociente de inteligência [Blair; Razza, 2007; Morrison et al, 2010], podendo predizer o desempenho em matemática e linguagem [Borella et al, 2010; Duncan et al, 2007].

Parte do que aprendemos requer que o cérebro formule um plano de ação baseado na experiência pregressa e, simultaneamente, se adapte a situação alvo; sendo 
VIII Congresso Brasileiro de Informática na Educação (CBIE 2019)

Anais do XXX Simpósio Brasileiro de Informática na Educação (SBIE 2019)

flexível para intermediar planos de acordo com as respostas das ações, sem perder o foco com as distrações do ambiente. Essas operações que controlam e regulam o processamento das informações são denominadas de funções executivas [Gazzaninga et al, 2006] e contemplam essencialmente os componentes de controle inibitório, memória de trabalho e flexibilidade cognitiva [Diamond, 2013]. O controle inibitório refere-se ao gerenciamento de responder ou não a um estímulo, o que significa inibir a propensão à resposta ou controlar a interferência, mantendo-se focado [Zelazo, 2016], similar à habilidade cognitiva requerida pelo jogo digital Fruit Ninja cuja mecânica consiste em cortar frutas que surgem na tela (responder) e não tocar nas bombas (inibição) que se misturam às frutas [Liu et al, 2015]. Já a memória de trabalho tem caráter transitório e refere-se à representação de informações relevantes à tarefa ou objetivo, recuperando as informações passadas ou relacionadas com experiências vivenciadas no tempo presente [Gazzaninga et al, 2006], por exemplo, ao calcular mentalmente a conta de um jantar. Envolvendo aspectos do controle inibitório e da memória de trabalho, a flexibilidade cognitiva refere-se à capacidade em alternar-se mentalmente entre situações, modificando e adaptando as ações conforme a execução da tarefa [Diamond, 2013].

A relação dos jogos digitais com o funcionamento cerebral vem sendo estudado para diversos fins. A revisão de Palaus et al (2017) identificou 116 estudos sobre mudanças estruturais ou funcionais no cérebro associadas ao uso de jogos digitais, com evidências neurocientíficas na melhora de habilidades visuoespaciais, atencionais e executivas. Os autores também identificaram falta de consenso sobre quais elementos dos jogos promovem a melhora nas habilidades cognitivas e por quanto tempo deve-se jogar um jogo para ocorrer o efeito de transferência (usar habilidade fora do ambiente de jogo); especialmente porque os jogos utilizados nos experimentos divergiam em suas características de design, levando a resultados controversos. As mesmas limitações foram discutidas na meta análise de Clark et al (2016) que enfatizou a importância de se discutir o efeito do design do jogo nas habilidades cognitivas, especialmente em relação aos jogos digitais utilizados na educação. Apesar disso, há evidências em revisões anteriores de tamanho de efeito grande $(n=72, d=0,61)$ e médio $(n=46, d=0,48)$, em estudos experimentais, de que os videogames melhoram a velocidade de processamento das informações [Powers et al, 2013].

Um jogo digital é o resultado da combinação de diversos constructos relativos à estética - cenário, narrativa, personagens, elementos sonoros e visuais que promovem a imersão no jogo e reações no jogador; à mecânica - componentes elementares que determinam as regras e o objetivo do jogo; e à dinâmica, resultado da interação do jogador com esses elementos, sua percepção da experiência [Novak, 2010]. Por exemplo, Prena et al (2018) identificaram que a presença de recompensas no jogo promove ativação da região hipocampal cortical implicando em melhor desempenho em memória nos jogadores em relação ao grupo controle, sem essa característica.

A recompensa é um dos elementos de mecânica do jogo, assim como outros componentes elementares de pontuação, tempo, vida, nível, fase e todas as ações e interações que o jogo permite [Novak, 2010], criando a engrenagem de fluxo e progressão motivadores ao jogador [Boyan; Sherry, 2011]. Também participam da construção de um jogo: tecnologia da plataforma - console, computador, dispositivo móvel; tipo de interação dos jogadores; forma de interação com a interface videogame, exergame, realidade aumentada, realidade virtual [Chandler, 2012]; propósito de construção - casual, sério - e área de aplicação [Connolly et al, 2012].

Outro termo comum característico dos jogos é o gênero, classificação a partir da 
VIII Congresso Brasileiro de Informática na Educação (CBIE 2019)

Anais do XXX Simpósio Brasileiro de Informática na Educação (SBIE 2019)

combinação do tema, interface, perspectiva do jogador e estratégia do jogo [Novak, 2010], sem uma definição consensual de quais mecânicas os compõem [Connoly et al, 2012]. Porquanto, a clareza nas características dos jogos digitais utilizados nas pesquisas em áreas aplicadas (educação, saúde) é essencial para investigação de evidências e achados mais contundentes [Clark et al, 2016; Palaus et al, 2017]. Sobre este contexto decorre o objetivo desta pesquisa de identificar quais mecânicas de jogos são utilizadas no estudo das funções executivas (avaliação ou treinamento), por meio da descrição dos jogos, sem considerar a classificação dada pelo gênero.

Para tal, a seção 2 apresentará o processo de construção da relação entre as variáveis e as relações identificadas, que serão discutidas na seção 3, findando com as considerações finais (seção 4) e as referências citadas neste estudo.

\section{Construindo a relação}

A base de dados para análise constituiu-se de 32 artigos científicos coletados em mapeamento sistemático anterior [Krause et al, 2018], com o termo de busca "executive function AND game". Cada artigo foi lido na íntegra por um pesquisador (limitação deste estudo) para extração dos dados. Por tratar-se de pesquisas multiáreas divergências terminológicas eram esperadas, admitindo-se sinônimos confrontando o referencial teórico com a descrição dos autores (base de dados). Para aqueles cuja relação mecânica do jogo e função executiva não estava explícita, a extração seguiu a metodologia PICO (população, intervenção, comparação, desfecho), sendo as mecânicas identificadas pela descrição do jogo, contexto e construção do experimento e as funções executivas analisando a questão de pesquisa, descrição dos testes de avaliação, perfil da amostra e desfecho. Informações ausentes ou não claras foram obtidas pela pesquisa de entorno (publicações anteriores e posteriores dos autores e referências citadas).

Dos 32 artigos analisados, excluiu-se um por apresentar versão atualizada no conjunto mapeado, resultando em 31 análises (artigos identificados por Idn, Tabela 2), dos quais $13 \%$ investigaram o funcionamento neural das funções executivas; $19 \%$ analisaram o impacto dos jogos no desempenho executivo; $22 \%$ relataram validação do jogo enquanto instrumento de avaliação executiva e $45 \%$ testaram o efeito do treinamento executivo por meio do jogo digital. A Tabela 1 apresenta a distribuição da faixa etária - crianças e adolescentes (29\%), jovens e adultos (45,2\%) e idosos (25,8\%) função executiva alvo, condição de saúde do grupo amostral e objetivo de pesquisa.

Tabela 1. Perfil geral das funções executivas alvo e objetivo de pesquisa

\begin{tabular}{|c|c|c|c|c|c|c|c|c|c|c|}
\hline \multirow[b]{2}{*}{$\begin{array}{c}\text { PERFIL } \\
\text { AMOSTRAL } \\
(n=31)\end{array}$} & \multicolumn{4}{|c|}{ FUNÇÃO EXECUTIVA } & \multicolumn{2}{|c|}{$\begin{array}{l}\text { CONDIÇÃO } \\
\text { SAÚDE }\end{array}$} & \multicolumn{4}{|c|}{ OBJETIVO DE PESQUISA } \\
\hline & 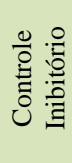 & 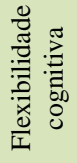 & 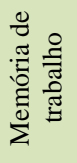 & 总: & 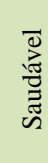 & 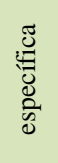 & 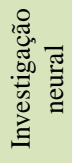 & 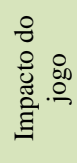 & 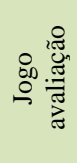 & 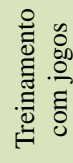 \\
\hline Criança $(\mathrm{N}=9)$ & 1 & 1 & 0 & 7 & 6 & 3 & 1 & 2 & 2 & 4 \\
\hline Adulto $(\mathrm{N}=14)$ & 2 & 3 & 2 & 7 & 7 & 7 & 3 & 3 & 3 & 5 \\
\hline Idosos (N=8) & 0 & 0 & 1 & 7 & 2 & 6 & 0 & 0 & 2 & 6 \\
\hline TOTAL & 3 & 4 & 3 & 21 & 15 & 16 & 4 & 5 & 7 & 15 \\
\hline
\end{tabular}

Tabela 2. Identificação dos artigos analisados $(n=31)$

\footnotetext{
ID $\quad$ REFERÊNCIA

Engelhardt, C. R., Hilgard, J., Bartholow, B. D. (2015). Acute exposure to difficult (but not violent) video games 1 dysregulates cognitive control. Computers in Human Behavior, v. 45, p. 85-92.
} 
VIII Congresso Brasileiro de Informática na Educação (CBIE 2019)

Anais do XXX Simpósio Brasileiro de Informática na Educação (SBIE 2019)

Dovis, S., Van der Oord, S., Wiers, R. W., Prins, P. J. (2015). Improving executive functioning in children with ADHD: Training multiple executive functions within the context of a computer game. A randomized double-blind placebo con-

2 trolled trial. PloS one, v. 10, n. 4.

Parong, J., Mayer, R. E., Fiorella, L., MacNamara, A., Homer, B. D., Plass, J. L. (2017). Learning executive function

3 skills by playing focused video games. Contemporary Educational Psychology, v. 51, p. 141-151.

Smittenaar, P., Rutledge, R. B., Zeidman, P., Adams, R. A., Brown, H., Lewis, G., Dolan, R. J. (2015). Proactive and

4 reactive response inhibition across the lifespan. PLoS One, v. 10, n. 10.

Klein, R. M., Hassan, T., Wilson, G., Ishigami, Y., Mulle, J. (2017). The AttentionTrip: A game-like tool for measuring

5 the networks of attention. Journal of neuroscience methods, v. 289, p. 99-109.

Yeh, C. W., Cherng, R. J., \& Chen, J. Y. (2013). The effect of visuospatial working memory training in elders: Devel-

6 opment and application of a brain fitness software. In 1st International Conference on Orange Technologies, p.270-273

Kretschmer, A., Altgassen, M., Rendell, P. G., Bölte, S. (2014). Prospective memory in adults with high-functioning autism spectrum disorders: Exploring effects of implementation intentions and retrospective memory load. Research in

7 developmental disabilities, v.35, n.11, p. 3108-3118.

McKenna, P.,et al (2014). One step at a time: Multimodal interfaces and children's executive functioning. In 4th Interna-

8 tional Conference on Development and Learning and on Epigenetic Robotics, p.421-425.

Mondéjar, T., Hervás, R., Johnson, E., Gutierrez, C., \& Latorre, J. M. (2016). Correlation between videogame mechanics

9 and executive functions through EEG analysis. Journal of biomedical informatics, 63, 131-140.

Gamito, P., Oliveira, J., Lopes, P., Morais, D., Brito, R., Cristóvão, S.,Rebelo, S. (2013). Executive functioning in addicts following health mobile cognitive stimulation: Evidence from alcohol and heroin patients. In Proceedings of the 7th

10 International Conference on Pervasive Computing Technologies for Healthcare, p.385-388. ICST

Avila-Pesantez, D., Rivera, L. A., Vaca-Cardenas, L., Aguayo, S., Zuñiga, L. (2018). Towards the improvement of ADHD children through augmented reality serious games: Preliminary results. In Global Engineering Education Confe-

11 rence,p.843-848.

Kayama, H., Okamoto, K., Nishiguchi, S., Yamada, M., Kuroda, T., Aoyama, T. (2014). Effect of a Kinect-based exercise game on improving executive cognitive performance in community-dwelling elderly: case control study. Journal of

12 medical Internet research, v.16, n.2.

Oei, A. C.,Patterson, M. D. (2014). Playing a puzzle video game with changing requirements improves executive fun-

13 tions. Computers in Human Behavior, v.37, p.216-228.

Anderson-Hanley, C., Barcelos, N. M., Zimmerman, E. A., Gillen, R. W., Dunnam, M., Cohen, B. D.,Maloney, M.

(2018). The Aerobic and Cognitive Exercise Study (ACES) for community-dwelling older adults with or at-risk for mild cognitive impairment (MCI): neuropsychological, neurobiological and neuroimaging outcomes of a randomized clinical

14 trial. Frontiers in aging neuroscience, v.10, n. 76

Schättin, A., Arner, R., Gennaro, F., de Bruin, E. D. (2016). Adaptations of prefrontal brain activity, executive functions, and gait in healthy elderly following exergame and balance training: a randomized-controlled study. Frontiers in aging

15 neuroscience, v. 8, n. 278.

Flynn, R. M., Richert, R. A., Staiano, A. E., Wartella, E., Calvert, S. L. (2014). Effects of exergame play on EF in children and adolescents at a summer camp for low income youth. Journal of educational and developmental psycho-

16 logy, v.4, n.1, p.209.

Kühn, S., Berna, F., Lüdtke, T., Gallinat, J., Moritz, S. (2018). Fighting depression: action video game play may reduce

17 rumination and increase subjective and objective cognition in depressed patients. Frontiers in psychology, v.9, n. 129.

Dassen, F. C., Houben, K., Van Breukelen, G. J., Jansen, A. (2018). Gamified working memory training in overweight

18 individuals reduces food intake but not body weight. Appetite, v. 124, p. 89-98.

Schoene, D., et al (2015). Interactive cognitive-motor step training improves cognitive risk factors of falling in older

19 adults-a randomized controlled trial. PLoS One, v. 10, n. 12

Shochat, G., Maoz, S., Stark-Inbar, A., Blumenfeld, B., Rand, D., Preminger, S., Sacher, Y. (2017, June). Motion-based virtual reality cognitive training targeting executive functions in acquired brain injury community-dwelling individuals:

20 A feasibility and initial efficacy pilot. In International Conference on Virtual Rehabilitation (ICVR), p.1-8. IEEE.

Goldstein, A., Déry, N., Pilgrim, M., Ioan, M., Becker, S. (2016). Stress and binge drinking: a toxic combination for the

21 teenage brain. Neuropsychologia, v. 90, p. 251-260.

Liu, Q., Zhu, X., Ziegler, A., Shi, J. (2015). The effects of inhibitory control training for preschoolers on reasoning

22 ability and neural activity. Scientific reports, v. 5

Colonna, A., Smith, A. B., Smith, S., VanDenEshof, K., Orgill, J., Gringras, P., Pal, D. K. (2018). The Effects of Sleep

23 on Emotional Target Detection Performance: A Novel iPad-Based Pediatric Game. Frontiers in psychology, v.9, n. 241.

Baniqued, L., Allen, M., Kranz, M. B., Johnson, K., Sipolins, A., Dickens, C.,Kramer, A. F. (2015). Working memory,

24 reasoning, and task switching training: transfer effects, limitations, and great expectations?. PLoS One, v. 10, n. 11

Vallejo, V., Wyss, P., Rampa, L., Mitache, A. V., Müri, R. M., Mosimann, U. P., Nef, T. (2017). Evaluation of a novel

25 Serious Game based assessment tool for patients with Alzheimer’s disease. PloS one, v. 12, n.5

Gray, S., Robertson, J., \& Rajendran, G. (2015). BrainQuest: an active smart phone game to enhance executive function.

26 In Proceedings of the 14th International Conference on Interaction Design and Children, p.59-68. ACM.

Burdea, G., Polistico, K., Krishnamoorthy, A., House, G., Rethage, D., Hundal, J., Pollack, S. (2015). Feasibility study

of the BrightBrainer ${ }^{\mathrm{TM}}$ integrative cognitive rehabilitation system for elderly with dementia. Disability and Rehabilita-

27 tion: Assistive Technology, v. 10, n. 5, p. 421-432.

Baranyi, R., Perndorfer, R., Lederer, N., Scholz, B., Grechenig, T. (2016). MyDailyRoutine-a serious game to support people suffering from a cerebral dysfunction. In International Conference on Serious Games and Applications for Health,

28

Tost, D., Pazzi, S., von Barnekow, A., Felix, E., Puricelli, S., Bottiroli, S. (2014). SmartAgeing: a 3D serious game for early detection of mild cognitive impairments. In Proceedings of the 8th International Conference on Pervasive Compu-

29 ting Technologies for Healthcare, p. 294-297. ICST 
VIII Congresso Brasileiro de Informática na Educação (CBIE 2019)

Anais do XXX Simpósio Brasileiro de Informática na Educação (SBIE 2019)

Li, B., Atyabi, A., Kim, M., Barney, E., Ahn, A. Y., Luo, Y., Mademtzi, M. (2018). Social Influences on Executive Functioning in Autism: Design of a Mobile Gaming Platform. In 2018 CHI Conference on Human Factors in Computing

30 Systems, p. 443.

Martínez-Pernía, D., Núñez-Huasaf, J., Del Blanco, Á., Ruiz-Tagle, A., Velásquez, J., Gomez, M., Slachevsky, A.

(2017). Using game authoring platforms to develop screen-based simulated functional assessments in persons with

31 executive dysfunction following traumatic brain injury. Journal of biomedical informatics, v.74, p. 71-84.

Majoritariamente os experimentos foram presenciais, exceto dois estudos realizados online ou por instalação do jogo no computador do pesquisado. A mensuração de desempenho centraram-se na diferença em pré e pós teste; efeito de transferência da habilidade adquirida em ambiente externo ao jogo; desempenho no jogo; experiência de mídia; usabilidade e aceitabilidade de uso da tecnologia, perfazendo 49 testes distintos incluindo avaliação neuropsicológica e ecológica (funcional), tarefas adaptadas de testes cognitivos, exame neurológico e questionários.

\subsection{O jogo é a variável manipulada ou não?}

Devido à escolha inicial de extração dos dados pela descrição dos experimentos, almejava-se encontrar manipulação das mecânicas para investigação de efeito, sendo possível a comparação de resultados advindos de design próximos. Este não foi o cenário encontrado no montante analisado, porém, quatro grupos puderam ser formados:

(a) Jogo A x Jogo A modificado: do tipo experimental exploratório, as pesquisas deste grupo centraram-se na identificação de recursos potenciais dos jogos para incremento nas funções executivas por meio de manipulação de elementos de um mesmo jogo digita. Exemplos de elementos da mecânica (recursos do jogo) manipulados: velocidade dos objetos, quantidade de objetos distradores, tempo de cada ciclo, restrição, pontuação, movimentação do avatar. Fazem parte deste grupo 25,8\% dos estudos ( $(\mathrm{Id}=1$ a 8$)$ e todos apresentaram grupo controle com atividade equivalente em relação às funções executivas testadas.

(b) Jogo A x Jogo B: experimental exploratório, mas diferentemente do anterior, neste tipo de experimento há confronto entre gêneros de jogo digital (ação, aventura, puzzle, ritmo/dança, tiro em primeira pessoa) e os componentes executivos requeridos por cada gênero (não necessariamente discutindo mecânicas). Este grupo é composto por 22,5\% dos estudos (Id = 9 ao 15), com validação através de grupo controle (exceto Id= 9 e 11 que o fizeram por variáveis dependentes).

(c) Jogo x Não jogo: tipo experimental comparativo, composto por pesquisas que compararam técnicas ou abordagens para melhorar a performance cognitiva ou motora, vislumbrando o jogo digital como uma abordagem de intervenção. Neste tipo de pesquisa os elementos do jogo não são alterados, apenas o contexto de intervenção, como ao comparar o impacto nas funções executivas de atividade física isolada em contraponto ao uso de exergame ou para identificar desempenho em teste cognitivo entre jogadores e não jogadores, contemplando $29 \%$ das pesquisas (Id = 16 a 24).

(d) Processo de design (D): de caráter descritivo, este grupo visa divulgação do processo de construção de um jogo digital voltado à avaliação ou treino executivo; sua importância está na divulgação e conhecimento do produto (jogo) e compreensão do desenvolvimento de requisitos e parâmetros. Participam deste perfil 22,5\% dos estudos (Id = 25 a 31), não sendo aplicável grupo controle, porém todos envolveram avaliação de viabilidade e/ou usabilidade, realizada por usuários ou especialistas.

O jogo digital não foi a variável foco da pesquisa para $I d=4,7,21$ e 23. Nestes o 
VIII Congresso Brasileiro de Informática na Educação (CBIE 2019)

Anais do XXX Simpósio Brasileiro de Informática na Educação (SBIE 2019)

jogo foi escolhido como um artefato para ativar áreas cerebrais das funções executivas supostamente prejudicadas devido a hábitos ou interação com outros componentes cognitivos. Por exemplo, Colonna et al $(2018, \mathrm{Id}=23)$ utilizaram o puzzle Pond Popper, para analisar o efeito da interrupção do sono em crianças $(n=30)$ na capacidade de atenção executiva, já Goldstein et al (2017, Id= 21) analisaram o impacto da combinação uso de álcool e estresse nas funções executivas de universitários. Apesar destes artigos não terem o jogo como variável manipulada, sua escolha para o experimento não foi arbitrária e por tal, foram mantidos para a análise de correlação.

\subsection{Função executiva $x$ mecânica de jogo digital}

Visando parear mecânicas dos jogos digitais com os componentes executivos, cada grupo (seção 2.1) foi analisado em relação às funções executivas alvo, descrição do jogo experimental, justificativa de escolha, componentes cognitivos avaliados pelos testes e desfecho. A Tabela 3 apresenta os termos considerados como sinônimos.

Tabela 3. Glossário de sinônimos adotados para pesquisa

\begin{tabular}{|c|c|c|}
\hline \multirow{4}{*}{ 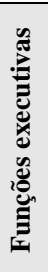 } & $\begin{array}{l}\text { Controle } \\
\text { inibitório (CI) }\end{array}$ & $\begin{array}{l}\text { controle inibitório, inibição, inibição da resposta, autocontrole, controle da atenção, controle de } \\
\text { interferência, atenção seletiva, autorregulação }\end{array}$ \\
\hline & $\begin{array}{l}\text { Memória de } \\
\text { trabalho (MT) }\end{array}$ & $\begin{array}{l}\text { memória de trabalho, memória sequencial, memória processual, memória operacional, memória } \\
\text { de trabalho viso espacial. }\end{array}$ \\
\hline & $\begin{array}{l}\text { Flexibilidade } \\
\text { cognitiva (FC) }\end{array}$ & $\begin{array}{l}\text { flexibilidade cognitiva, criatividade, alternância entre tarefas, flexibilidade de atenção, fluidez de } \\
\text { pensamento, pensamento fluído, deslocamento, multitarefa, dupla-tarefa. }\end{array}$ \\
\hline & $\begin{array}{l}\text { Função } \\
\text { executiva (FE) }\end{array}$ & $\begin{array}{l}\text { funções executivas gerais ou que incluem todos os componentes, } \\
\text { controle executivo, planejamento, estratégia, lógica, raciocínio }\end{array}$ \\
\hline \multirow{2}{*}{ 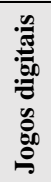 } & Videogame & $\begin{array}{l}\text { videogame, jogo eletrônico, jogo digital - com ou sem mundo virtual, simulação; jogos cujo } \\
\text { movimento corporal do jogador restringe-se ao digital/manual }\end{array}$ \\
\hline & $\begin{array}{l}\text { Exergame } \\
\text { EG) }\end{array}$ & $\begin{array}{l}\text { exergame, jogo ativo, videogame interativo - com ou sem mundo virtual, exergame para MMSS } \\
\text { (membro superior), jogo com realidade aumentada (mas descrito como movimento corporal } \\
\text { amplo para ações do jogador na projeção em tela) }\end{array}$ \\
\hline
\end{tabular}

Os jogos são compostos de tarefas ou ações direcionadas ao jogador para atingir os objetivos do jogo. A descrição feita pelos autores embasou as mecânicas extraídas (Mn), identificadas e aglutinadas por verbos. Por exemplo, "saltar sobre lírios”, "pular buracos no caminho" ou "desviar de bombas" foi considerado como única mecânica "saltar, pular, desviar obstáculos" (M6) relacionada com o grupo que exige resposta rápida a um estímulo, característica típica do controle inibitório. A Tabela 4 reúne as 22 mecânicas identificadas, das quais 15 foram associadas ao controle inibitório; 10 com memória de trabalho; 10 à flexibilidade cognitiva e 11 atribuídas ao envolvimento de todas as funções executivas (sem estratificação por componentes).

Tabela 4. Relação entre funções executivas e mecânicas de jogos digitais

\begin{tabular}{|c|c|c|c|}
\hline & Ações/Mecânicas associadas à função executiva & Qtde & Id Artigos \\
\hline 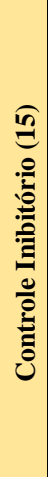 & $\begin{array}{l}\text { M1: Reagir sob ação rápida } \\
\text { M2: Selecionar, escolher entre os distradores (tomada de decisão) } \\
\text { M3; Mirar, atirar, destruir } \\
\text { M4: Agir e reagir conforme regra ativa ou inibitória (se x faço, se y não faço) } \\
\text { M5: Suspender ação por período de tempo e acioná-la posteriormente (tempo } \\
\text { de suspensão maior que o tempo de reação) - ação oportuna } \\
\text { M6: Saltar, pular, desviar obstáculos } \\
\text { M7: Navegar desviando de obstáculos ou distradores enquanto captura alvos } \\
\text { M8: Alternar ações, movimentos, padrões } \\
\text { M9: Resistir a opções de domínio para executar tarefas necessárias (resistir à } \\
\text { tentação de repetir ou fazer aquele percebido como mais fácil) } \\
\text { M10: Repetir movimentos do avatar, imitar sequência motora } \\
\text { M11: Ser induzido a manter ações e repentinamente ter de suspendê-la por um }\end{array}$ & $\begin{array}{l}9 \\
6 \\
4 \\
4 \\
3 \\
2 \\
2 \\
2 \\
1 \\
1 \\
1 \\
1\end{array}$ & $\begin{array}{l}1,2,4,9,19,15,22,25,27 \\
11,15,19,23,25,30 \\
1,5,9,27 \\
4,15,22,25 \\
4,25,30 \\
1,5 \\
2,5 \\
19,25 \\
26 \\
9 \\
2\end{array}$ \\
\hline
\end{tabular}


VIII Congresso Brasileiro de Informática na Educação (CBIE 2019)

Anais do XXX Simpósio Brasileiro de Informática na Educação (SBIE 2019)

\begin{tabular}{|c|c|c|c|}
\hline & $\begin{array}{l}\text { tempo, voltando a ação anterior induzida } \\
\text { M12: Comutar lados do corpo (correspondência de ação com parte do corpo) } \\
\text { M13: Parear informação (tem/não tem), observar diferenças entre telas (similar } \\
\text { ao jogo dos sete erros) } \\
\text { M14: Recordar o que precisa ser selecionado (itens) } \\
\text { M15: Planejar as ações antes de executá-las }\end{array}$ & $\begin{array}{l}1 \\
1 \\
\end{array}$ & $\begin{array}{l}27 \\
11 \\
11 \\
13 \\
\end{array}$ \\
\hline 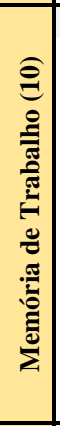 & $\begin{array}{l}\text { M16: Recordar sequência de tarefas (passos, objetivos, missões) a cumprir } \\
\text { M14: Recordar o que precisa ser selecionado (itens) } \\
\text { M15: Planejar as ações antes de executá-las (descrever sequência do plano) } \\
\text { M13: Parear informação (tem/não tem), observar diferenças entre telas (similar } \\
\text { ao jogo dos sete erros) } \\
\text { M17: Alternar ações conforme regras modificáveis ou conflitantes (inversas) } \\
\text { M18: Resolver e descobrir enigmas ou padrões lógicos } \\
\text { M19: Ordenação por correspondência a regra que altera (invertem) a ordem em } \\
\text { determinados ciclos (25\% dos ensaios) } \\
\text { M8: Alternar ações, movimentos, padrões } \\
\text { M2: Selecionar, escolher entre os distradores (tomada de decisão) } \\
\text { M10: Repetir movimentos do avatar, imitar sequência motora }\end{array}$ & $\begin{array}{l}7 \\
5 \\
1\end{array}$ & $\begin{array}{l}6,7,9,18,24,26,29 \\
15,18,24,29,30 \\
2 \\
24 \\
18 \\
9 \\
18 \\
9 \\
21 \\
9 \\
\end{array}$ \\
\hline 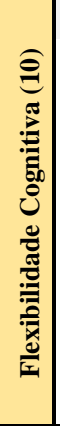 & $\begin{array}{l}\text { M15: Planejar as ações antes de executá-las (buscar melhor solução, caminho) } \\
\text { M17: Alternar ações conforme regras modificáveis ou conflitantes (inversas) } \\
\text { M18: Resolver e descobrir enigmas ou padrões lógicos } \\
\text { M8: Alternar ações, movimentos, padrões } \\
\text { M20: Rotacionar peça mentalmente e encaixar (similar Tetris, quebra-cabeça) } \\
\text { M14: Recordar o que precisa ser selecionado (itens) } \\
\text { M21: Comutar entre papéis de jogador (diferentes atribuições, regras, poder) } \\
\text { M11: Ser induzido a manter ações e repentinamente ter de suspendê-la por um } \\
\text { tempo, voltando a ação anterior induzida } \\
\text { M13: Parear informação (tem/não tem), observar diferenças entre telas (similar } \\
\text { ao jogo dos sete erros) } \\
\text { M19: Ordenar por correspondência a regras que se alteram (invertem) em } \\
\text { determinados ciclos (25\% dos ensaios) }\end{array}$ & $\begin{array}{l}5 \\
4 \\
4 \\
3 \\
2 \\
1 \\
1\end{array}$ & \begin{tabular}{|l}
$8,13,15,19,24$ \\
$2,3,19,24$ \\
$9,12,24,30$ \\
$3,9,19$ \\
15,19 \\
3 \\
26 \\
2 \\
15 \\
2
\end{tabular} \\
\hline 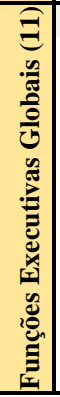 & $\begin{array}{l}\text { M22: Executar tarefa funcional cotidiana constituída em etapas (sequenciais) } \\
\text { M18: Resolver e descobrir enigmas ou padrões lógicos } \\
\text { M15: Planejar as ações antes de executá-las (buscar melhor solução, caminho) } \\
\text { M1: Reagir sob ação rápida } \\
\text { M7: Navegar desviando de obstáculos ou distradores enquanto captura alvos } \\
\text { M14: Recordar o que precisa ser selecionado (itens) } \\
\text { M2: Selecionar, escolher entre os distradores e o alvo (tomada de decisão) } \\
\text { M6: Saltar, pular desviar obstáculos } \\
\text { M8: Alternar ações, movimentos, padrões } \\
\text { M16: Recordar a sequência de tarefas (passos, objetivos, missões) a cumprir } \\
\text { M12: Comutar lados do corpo (correspondência de ação com parte do corpo) }\end{array}$ & $\begin{array}{l}5 \\
3 \\
3 \\
2 \\
2 \\
1 \\
1 \\
1\end{array}$ & \begin{tabular}{|l|}
$10,20,28,29,31$ \\
$9,24,27$ \\
$1,13,17$ \\
16,17 \\
5,14 \\
27 \\
14 \\
17 \\
25 \\
20,28 \\
27 \\
\end{tabular} \\
\hline
\end{tabular}

\section{Discussão}

As pesquisas abrangeram todas as faixas etárias, com enfoques tanto na avaliação $(22,5 \%)$ quanto no treinamento $(48,4 \%)$ das habilidades executivas. O interesse em criar jogos para aprimoramento dessas habilidades em crianças e adolescentes representou um terço dos estudos (n=9). Este cenário concorda com Zelazo et al (2016) que alertam para a importância de programas e ações que promovam as funções executivas já no início da vida escolar, com intuito de melhorar o desempenho (facilidade na compreensão), reduzir comportamentos impulsivos, aumentar a capacidade atencional e de resolução de problemas. A revisão de Girard et al (2013) apresenta várias evidências do benefício do uso de jogos digitais para aprendizagem e Mayer (2019) enfoca a importância dos jogos para desenvolvimento de habilidades cognitivas no âmbito escolar. Neste levantamento 22 mecânicas de jogos digitais foram usadas para o desenvolvimento de funções executivas de crianças e adolescentes e não houve diferenciação das mecânicas por faixa etária, exceto para os jogos de simulação que envolveu atividades cotidianas (M22) para avaliação e treinamento de idosos. 
VIII Congresso Brasileiro de Informática na Educação (CBIE 2019)

Anais do XXX Simpósio Brasileiro de Informática na Educação (SBIE 2019)

A pesquisa de McKenna et al (2014, Id= 8), por exemplo, comparou o desempenho das crianças no jogo Slippy's Adventure (M15: planejar ação antes de executá-la, buscar a melhor solução, descrever sequência do plano), construído com base no teste de flexibilidade cognitiva Torre de Hanoy. Para tal, utilizaram a versão do jogo em videogame e exergame e descobriram que, diferentemente do que ocorre com idosos, a versão exergame gerou sobrecarga cognitiva, sendo a perfomance melhor no modo videogame. Já Mondejár et al (2016, Id=9) correlacionaram as funções executivas ativadas ao jogar diferentes tipos de jogos e identificaram que resolver enigmas lógicos para avançar no jogo (M18) apresentou alta correlação dos exames por imagem com o desempenho no teste de Stroop, que mensura o controle inibitório.

Exceto pelas mecânicas M3, M4 e M5, relacionadas ao controle inibitório, descritas respectivamente como "mirar, atirar, destruir'; "agir e reagir conforme regra ativa ou inibitória (se x faço, se y não faço)" e "suspender ação por período de tempo (maior que o tempo de reação) e acioná-la posteriormente (ação oportuna)"; as mecânicas foram correlacionadas a mais de um componente, em conformidade com a dificuldade em isolar os componentes executivos funcionalmente [Diamond, 2013]. Apesar disso, o levantamento dos dados pode sublinhar que as mecânicas (ações impostas ao jogador) de alternar ações, movimentos e padrões (M8); recordar o que precisa ser selecionado (itens) para uso subsequente (M14) e planejar ações antes de executá-las - descrever sequência do plano ou buscar a melhor solução - (M15), envolvem todos os componentes executivos, sendo citadas tanto isoladamente quanto agrupadas por $51,6 \%$ dos artigos analisados, apontando um forte indicador relacional.

Outros indicadores relacionais entre funções executivas e mecânicas - regras para atuação e progressão no jogo - relatados com frequência foram: "reagir sob ação rápida" (M1) com controle inibitório; "recordar a sequência de tarefas (passos, objetivos, missões) a cumprir” (M16) com a memória de trabalho e "planejar as ações antes de executá-las” (M15) com flexibilidade cognitiva.

A mecânica é o elemento vivo de um jogo que delimita e orienta as ações do jogador [Novak, 2010] e por tal, influencia nas habilidades cognitivas necessárias para execução das tarefas. Os elementos como tempo, pontuação e velocidade dos objetos impõem o ritmo ao jogador e essa atmosfera cria o campo ideal para o exercício das funções executivas: desviando de obstáculos e destruindo inimigos (controle inibitório), mantendo-se atento aos passos a serem seguidos (memória de trabalho) e planejando estratégias (flexibilidade cognitiva) para atingir o alvo e progredir de fase.

Apesar da nomenclatura abstrata, elementos de design de jogos que favorecem o aprendizado de habilidades cognitivas, citados por Qian et al (2016), também foram encontrados nas mecânicas descritas para funções executivas desta revisão. Arnab et al (2014) desenvolveram uma ferramenta de análise de jogos digitais para educação correlacionando mecânicas de aprendizagem com mecânicas de jogos, dividindo-as em mecânicas abstratas (diversão, desafio, penalidades, chances, competição, etc) e concretas (simulação, questões/respostas, seleção, tutorial, movimento, feedback rápido, etc.). No entanto, essas descrições não foram suficientes para compreensão das características do jogo (mecânicas) e componentes cognitivos, principalmente os componentes executivos, devido à necessidade de caracterização mais específica. $\mathrm{O}$ mesmo foi observado nas revisões e meta-análises pesquisadas no entorno dos artigos revisados por esta pesquisa, o que motivou o objetivo desta pesquisa em correlacionar, 
VIII Congresso Brasileiro de Informática na Educação (CBIE 2019)

Anais do XXX Simpósio Brasileiro de Informática na Educação (SBIE 2019)

de forma concreta, mecânicas de jogos e componentes executivos.

\section{Considerações finais}

Independentemente da classificação adotada, todo jogo digital é composto por tarefas (ações) que estão direcionadas a um ou mais objetivos, e é neste ponto que os jogos digitais interceptam as funções executivas. Existe interesse na comunidade científica em compreender quais os mecanismos que produzem o efeito benéfico na cognição e os experimentos têm adotado diversos tipos de jogos nesta investigação. No entanto, saber o nome ou gênero do jogo é insuficiente para essa associação, pois um jogo de puzzle pode conter mecânicas de um jogo de ação e vice-versa, impactando diferentemente nas habilidades executivas exercitadas.

Esta pesquisa analisou os artigos enfocando as características dos jogos digitais utilizados em experimentos envolvendo a temática de funções executivas, especificamente nas ações delimitadas para o jogador. A análise das ações propostas aos jogadores possibilitou definir 22 mecânicas de jogos utilizadas para avaliação e treinamento de habilidades executivas. Compreender melhor as mecânicas presentes nos jogos digitais e como se relacionam com os componentes das funções executivas, acredita-se contribuir para avaliação do educador de qual jogo digital relaciona-se melhor com a função executiva que deseja fomentar nos alunos. Complementarmente, jogos digitais educacionais poderão ser avaliados com vistas ao espectro das funções executivas que promovem, obtendo-se conjunto de recursos concentrados em poucos jogos. Iniciativas estas que reafirmam a importância e utilidade das funções executivas em jogos digitais. Por fim, almeja-se que esta pesquisa se amplie, para que outras mecânicas possam ser identificadas, confrontadas e manipuladas; estabelecendo relações significativas entre mecânica de jogos e cognição e clarificando questões em aberto sobre quais características de jogos produzem efeitos potentes nas habilidades executivas e que se transponham para os diversos âmbitos fora do ambiente de jogo, inclusive educacional.

\section{Agradecimentos}

Este trabalho foi realizado com apoio do Programa de Bolsas de Monitoria de Pós-GraduaçãoPROMOP da UDESC a qual agradecemos pelo fomento e incentivo à pesquisa. Agradecemos o apoio financeiro da FAPESC, Edital FAPESC/CNPQ Nº6/2016, T.O. N ${ }^{\circ}: 2017 T R 1755$ Ambientes Inteligentes Educacionais com Integração de Técnicas Learning Analytics e Gamificação e FAPESC Edital N 01/2016 - Termo de Outorga $n^{0} 2017 T R 649$ - Processo 531/2017- Apoio a Grupos de Pesquisa da Universidade do Estado de Santa Catarina (UDESC) e ao CNPq pela bolsa produtividade DT-2.

\section{Referências}

Arnab, S., Lim, T., Carvalho, M. B., Bellotti, F., De Freitas, S., Louchart, S., ... \& De Gloria, A. (2015). "Mapping learning and game mechanics for serious games analysis". British Journal of Educational Technology, v. 46, n. 2, p. 391-411.

Blair, C., Razza, R. P. (2007). "Relating effortful control, executive function, and false belief understanding to emerging math and literacy ability in kindergarten”. Child development, v. 78, n.2, p. 647-663.

Boyan, A., Sherry, J. L.(2011). “The challenge in creating games for education”: Aligning mental models with game models. Child development perspectives, v. 5, n.2, p. 82-87.

Chandler, H. M. (2009). Manual de produção de jogos digitais. Bookman Editora.

Clark, D. B.; Tanner-Smith, E. E.; Killingdworth, S. (2016). "Digital games, design, and 
VIII Congresso Brasileiro de Informática na Educação (CBIE 2019)

Anais do XXX Simpósio Brasileiro de Informática na Educação (SBIE 2019)

learning”: A systematic review and meta-analysis. Review of educational research, v. 86, n. 1, p. 79-122.

Connolly, T. M., Boyle, E. A., MacArthur, E., Hainey, T., \& Boyle, J. M. (2012). “A systematic literature review of empirical evidence on computer games and serious games". Computers and Education, v. 59, n. 2, p. 661-686.

Cox, K. K.; Bittencourt, R. A. (2017) "Estudo bibliográfico sobre o processo de construção de jogos digitais”: a necessidade de sinergia entre o educar e o divertir. Revista Brasileira de Informática na Educação - RBIE, v.25, n.1, p.16-43.

Diamond, A. (2013) "Executive functions”. Annual review of psychology, v. 64, p.135-168.

Engelhardt, C. R., Hilgard, J., \& Bartholow, B. D. (2015). “Acute exposure to difficult (but not violent) video games dysregulates cognitive control”. Computers in Human Behavior, v.45, p. 85-92.

Gazzaninga, Michael S.; Ivry, Richard B.; Mangun, George Ronald. (2006) "Neurociência cognitiva”: a biologia da mente. Artmed.

Girard, C.; Ecalle, J.; Magman, A. (2013). "Serious games as new educational tools": how effective are they? A meta-analysis of recent studies. Journal of Computer Assisted Learning, v. 29, n. 3, p. 207-219.

Goldstein, A., Déry, N., Pilgrim, M., Ioan, M., \& Becker, S. (2016). "Stress and binge drinking”: a toxic combination for the teenage brain. Neuropsychologia, v. 90, p. 251-260.

Katz, N. (2008). "Neurociência, Reabilitação Cognitiva e Modelos de Intervenção Em Terapia Ocupacional”. Grupo Gen-Livraria Santos Editora.

Krause, K. K. G.; Gasparini, I. Hounsell, M. da S. (2018) “Aplicações de jogos digitais nas funções executivas”: um mapeamento sistemático da literatura. In: Proceedings of the XVII Brazilian Symposium on Computer Games and Digital Entertainment, p. 54-62.

Kuhn, Deanna. (2006). "Do cognitive changes accompany developments in the adolescent brain?”. Perspectives on Psychological Science, v. 1, n. 1, p. 59-67.

Liu, Q., Zhu, X., Ziegler, A., \& Shi, J. (2015). “The effects of inhibitory control training for preschoolers on reasoning ability and neural activity.” Scientific reports, v. 5.

Mattar, João. (2010). "Games em educação”: como os nativos digitais aprendem. São Paulo: Pearson Prentice Hall.

Mayer, Richard E. (2019). “Computer games in education”. Annual review of psychology, v. 70, p. 531-549.

McKenna, P., Lemon, O., Corley, M., Boa, D., \& Rajendran, G. (2014). “One step at a time”: Multimodal interfaces and children's executive functioning. In 4th International Conference on Development and Learning and on Epigenetic Robotics, p. 421-425, IEEE.

Novak, J. (2010). “Desenvolvimento de games”. São Paulo: Cengage Learning.

Palaus, M., Marron, E. M., Viejo-Sobera, R., \& Redolar-Ripoll, D. (2017). "Neural basis of video gaming”: A systematic review. Frontiers in human neuroscience, v. 11, p. 248.

Powers, K. L., Brooks, P. J., Aldrich, N. J., Palladino, M. A., \& Alfieri, L. (2013). "Effects of video-game play on information processing”: a meta-analytic investigation. Psychonomic bulletin \& review, v. 20, n. 6, p. 1055-1079.

Prena, K., Reed, A., Weaver, A. J., \& Newman, S. D. (2018). "Game Mechanics Matter”: Differences in Video Game Conditions Influence Memory Performance. Communication Research Reports, v. 35, n. 3, p. 222-231.

Qian, M., \& Clark, K. R. (2016). "Game-based Learning and 21st century skills”: A review of recent research. Computers in Human Behavior, v. 63, p. 50-58.

Zelazo, P. D., Blair, C. B., Willoughby, M. T. (2016). “Executive Function”: Implications for Education. NCER 2017-2000. National Center for Education Research. 